\title{
Evolución del deterioro cognitivo y el nivel de dependencia en pacientes mayores de 65 años ingresados en un hospital de agudos: relación con las variables sociodemográficas
}

\author{
Mª José Calero-García y Alfonso J. Cruz Lendínez \\ Universidad de Jaén (España)
}

\begin{abstract}
El primer objetivo de este trabajo es establecer y estudiar la evolución de las etapas de deterioro cognitivo y los niveles de dependencia en pacientes mayores de 65 años, ingresados en un hospital de agudos, así como la relación entre estos dos factores y las diferentes variables demográficas. Los resultados muestran que el nivel de dependencia experimenta una caída repentina en el momento de la admisión, que evoluciona hacia una recuperación leve en el momento del alta, continuando esta recuperación en el domicilio posteriormente al alta, pero sin llegar a los niveles independentistas antes de la admisión. Además, se encontraron diferencias significativas en cuanto a edad, estado civil y nivel educativo. De una manera general, nuestros resultados proporcionan evidencia sobre el hecho de que las personas especialmente propensas al aumento de la dependencia funcional y el deterioro cognitivo severo durante el ingreso hospitalario son los ancianos hombres, mayores de 80 años de edad, sin estudios y viudos.
\end{abstract}

Palabras clave: Deterioro cognitivo, dependencia funcional, hospitalización, envejecimiento.

Cognitive and functional impairment of hospitalized patients over 65 years of age. The first objective of this research is to establish and study how the different stages of cognitive impairment and the levels of dependence evolve in patients over 65 years of age, admitted to an acute care hospital, as well as the relationship between these factors and the different social and demographical variables. The results show that the level of dependence decreases suddenly at the time of admission and undergoes a slight recovery at the time of discharge. Although this recovery continues at home after discharge, patients do not get the same level of independence that they used to have before admission. In addition, significant differences in terms of age, marital status and education level were found. In general, our results show that elderly men over 80 years of age, without no education and widowers are more likely to suffer from severe cognitive impairment and be more functionally dependent when admitted to hospital.

Keywords: Cognitive impairment, functional dependence, hospitalization, aging.

Correspondencia: $\mathrm{M}^{\mathrm{a}}$ José Calero-García. Facultad de Ciencias de la Salud. Universidad de Jaén. Campus Las Lagunillas. C.P.: 23071. Jaén (España).E-mail: mjcalero@ujaen.es 
El impacto de la enfermedad sobre el estado funcional de una persona mayor es la característica fundamental que los diferencia de los pacientes de otros grupos de edad. Dado que la funcionalidad es el atributo que debe ser preservado en los ancianos, la mejor estrategia de atención es evitar su pérdida, en lugar de recuperarla una vez perdida. En consecuencia, hay un cambio en el enfoque de los pacientes geriátricos; desde el tratamiento de una enfermedad y la recuperación de una función perdida, a la prevención del deterioro de la funcionalidad en geriátrica y gerontológica, procurando un envejecimiento más saludable. Dentro de este contexto hemos modelado un concepto de fragilidad, que abarca los aspectos de deterioro cognitivo, la dependencia, la pérdida de las reservas fisiológicas, la desconexión del entorno, enfermedades crónicas o el envejecimiento acelerado. Por lo tanto, buscamos la relación entre los factores clínicos y los mecanismos biológicos que producen debilidad e incapacidad, tanto entendida como la restricción o ausencia de la capacidad para realizar una actividad, como las alteraciones sobre el desempeño de la actividad normal. Esta incapacidad y debilidad crean problemas de adaptación que limitan el rendimiento individual y aumentan su dependencia funcional, o la necesidad de asistencia y el apoyo de de terceros para llevar a cabo estas actividades y tareas (Elipe, 2006). La funcionalidad ha sido definida como la capacidad del individuo para adaptarse a la subsistencia diaria a pesar de sufrir una discapacidad física o psíquica (Bravo, 2008). La funcionalidad puede ser evaluada de acuerdo con la capacidad de realizar actividades de la vida diaria. Algunos modelos teóricos consideran el deterioro cognitivo como factor inicial, o un factor para predecir la fragilidad, entendida como la modificación de las funciones mentales que el envejecimiento conlleva-en mayor o menor medida-y se refleja en un ritmo más lento en la solución de problemas abstractos, dificultad para mantener atención a los estímulos simultáneos, el retraso en el nombramiento y mantenimiento de vocabulario y la dificultad de almacenar nueva información, aunque no de la adquisición a través de la experiencia (Varela, Chávez, Gálvez y Méndez, 2004). Este declive cognitivo contribuirá a la fragilidad, tanto de forma directa, con la disminución de la condición física y la reducción de la funcionalidad, e indirectamente, la causa y la condición de baja ingesta de alimentos (Macías, Guerrero y Hernández, 2008).

La definición de la situación de dependencia, aprobado por el Consejo de Europa en septiembre de 1998, se centra en tres factores concurrentes: en primer lugar, la existencia de una limitación física, psíquica o intelectual que obstaculiza las capacidades de las personas, en segundo lugar, su incapacidad para realizar por sí mismas las actividades de la vida diaria, y en tercer lugar, la necesidad de asistencia o apoyo de otra persona para su propio cuidado. Aunque existe una relación entre la estancia anterior en una residencia y la dependencia, el aumento de la dependencia no parece estar asociado a la estancia en residencias de cuidado geriátrico (Calero, Navarro, Gómez, López y García, 2008), pero sí, a la hospitalización y sus factores concomitantes 
(Parada et al., 2009). Hay una tendencia real a la cronicidad y la discapacidad en el anciano hospitalizado, y tras el alta hay una gran posibilidad de pérdida funcional y mental que aumentará, la mortalidad, la institucionalización y la necesidad y el uso de servicios sociales y recursos sanitarios (Calero et al., 2008). De hecho, la hospitalización es para una persona de edad, independientemente de la causa de la admisión, un factor de riesgo. Incluso la OMS, en 1974, incluyó en su definición de los factores de riesgo del "período inmediato después del alta hospitalaria" (Canga y Narvaiza, 2006).

Para las personas mayores, la hospitalización significa la limitación de espacio de vida y una reducción de la movilidad que contribuye a la disminución cognitiva. Por lo tanto, las repercusiones negativas que el deterioro funcional tiene en la calidad del paciente de edad avanzada de la vida durante la hospitalización, hace que posteriormente el anciano sufra un envejecimiento menos saludable, agravado por la disminución de las capacidades cognitivas, que conlleva un importante deterioro de las relaciones sociales, fomenta el aislamiento y la depresión, aumenta la dependencia funcional y tiene un coste económico y social alto; y en casos extremos puede incluso causar la muerte (Delgado et al, 2009; Feng, Sherer, Tan, Chan y Fong, 2010). Los factores moduladores de una situación de declive funcional y cognitivo durante la hospitalización de los pacientes ancianos no están suficientemente estudiados (Varela, Chávez, Herrera, Ortiz y Chigne, 2004; Varela et al., 2004). Una evaluación integral del paciente anciano hospitalizado debe incluir tanto la capacidad de ejecutar las tareas intelectuales y físicas (Macías et al., 2008). De acuerdo a los hechos arriba mencionados, la evaluación del estado de funcionalidad es indispensable en la evaluación geriátrica integral de la persona mayor (CGA) como un predictor de la salud y las condiciones de recuperación posterior de otras patologías.

La identificación de una persona de edad avanzada en riesgo de manifestar condiciones adversas, como disminución de la funcionalidad y por consiguiente un aumento de la dependencia es todavía un problema. Se recomienda tener en cuenta como factores de riesgo en personas de edad avanzada: la vejez (más de 80 años de edad), hospitalización reciente, las caídas, los cambios en la movilidad y el equilibrio, debilidad muscular y el sedentarismo, la comorbilidad, las enfermedades cardiovasculares y mentales, las circunstancias sociales adversas y la polifarmacia (Martín, Gorroñogoitia, Gómez, Baztán y Abianza, 2010). También es importante mencionar que la tasa de incidencia en este tipo de problemas es mayor en las mujeres que en los hombres (Villón y Pecho, 2003). El aumento de dependencia funcional experimentada por pacientes ancianos hospitalizados se ha relacionado con decúbito, bajo nivel de interacción social debido a períodos prolongados de reposo en cama, y una estancia hospitalaria más prolongada (Delgado et al., 2009; Gutiérrez, Domínguez y Solano, 1999). En relación con el descanso y la duración de la estancia hospitalaria, en un estudio sobre la hospitalización de los pacientes de más de 65 años de edad con fractura de hueso de la 
cadera, se demostró que había un fuerte vínculo entre los días previos a la cirugía, en la que los pacientes son inactivos, y un mayor declive funcional y cognitivo (Calero, Ortega, Navarro, Díaz y Calero, 2011).

Pedone, Ercolani, Catani y Maggio (2005) señalan que en pacientes de mayor edad, el deterioro cognitivo al ingreso y durante la hospitalización se asociaron a un mayor riesgo de pérdida funcional, independientemente de la edad, sexo, comorbilidad, polifarmacia y la discapacidad en admisión. Por otro lado, Covinsky y Palmer (2003) observaron que la tasa de pérdida de capacidad funcional en personas de edad avanzada fue mayor en los pacientes de más edad, de una pérdida de $23 \%$ en pacientes entre 70 y 75 , a una pérdida de $63 \%$ para los pacientes más de 90 y de $50 \%$ para los pacientes de 85 años de edad. Varela, Chávez, Gálvez y Méndez (2004), en un estudio de investigación sobre la evaluación geriátrica integral (CGA) en las personas de edad avanzada hospitalizados en el Perú, encontraron una asociación significativa entre el deterioro cognitivo, nivel educativo, deterioro auditivo, falta de movilidad, incontinencia fecal y urinaria, la desnutrición, la depresión y el nivel de funcionalidad.

Como se ha demostrado, hay dos factores, la edad y el estado cognitivo, cuya interacción se vuelve relevante para analizar la calidad de vida y el nivel de dependencia de la persona mayor. Al analizar su interacción, a pesar de las diferencias existentes debido a la edad, hay muchas más diferencias debidas al estado cognitivo (Calero y Navarro, 2011). Como consecuencia de todo lo expuesto anteriormente, el primer objetivo de este trabajo fue establecer el deterioro cognitivo y en el nivel de dependencia en pacientes mayores de 65 años de edad, que han sufrido un periodo de hospitalización en un hospital de agudos. Además de estudiar la relación entre este deterioro y las diferentes variables demográficas.

\section{MÉTODO}

\section{Participantes}

La muestra estaba compuesta por 259 participantes que ingresaron en el Hospital de Neurotraumatológico de Jaén, España. Los criterios de inclusión fueron: tener 65 años o más, la duración de la estancia hospitalaria de más de 5 días, no sufrir una enfermedad aguda incapacitante (AIS), y no estar en condición terminal. $78.4 \%$ de los participantes eran mujeres y $21.6 \%$ hombres, estas cifras son comparables a otros estudios de investigación de este grupo de población (Baztán, Fernández-Alonso, Aguado y Socorro, 2004; Cruz et al., 2010; Elipe, 2006; Navarro, Calero, López, Gómez y García-Calero, 2008).

Por lo que respecta a su estado civil: $44 \%$ casados, $47.5 \%$ eran viudos y el $8.5 \%$ eran solteros. En cuanto a su nivel educativo: el $63.7 \%$ no tenía ningún tipo de educación, el $36.3 \%$ (79) tenían algún tipo de educación y sólo el $1.5 \%$ de los 
participantes tenía educación superior. Las edades oscilaron entre 65 y 105 años $(M=80.37, S D=8.352)$. $50.2 \%$ de los participantes pertenecían al grupo de entre 65 y 80 años de edad $(M=73.65, S D=4.775)$ y el $49.8 \%$ tenía entre 81 y 105 años de edad $(M=87.14$. $D T=5.062)$. En ambos grupos de edad había un mayor número de mujeres.

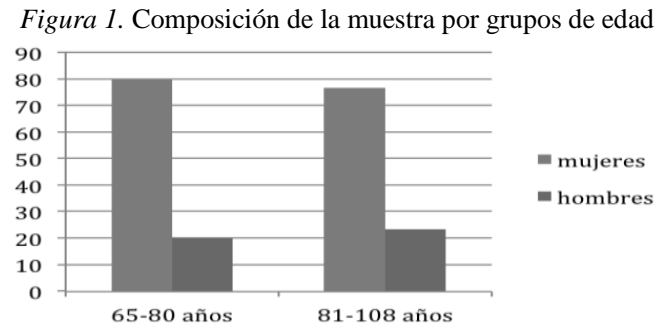

\section{Instrumentos}

Los datos sociodemográficos se obtuvo mediante una entrevista semiestructurada diseñada ad hoc. Se utilizó el Índice de Barthel (Granger, Dewis, versión Peters, Sherwood y Barrte de 1979, adaptado al español por Damián Cid, 1997) para medir el nivel de dependencia. Shah, Vanclay y Cooper (1989) sugirió un índice de calificación en la que 0 a 60 puntos se considera como dependencia severa, 61 a 90, como dependencia moderada, 91 a 99 como baja dependencia y 100 como la independencia completa. Se utilizó la Escala de Lawton y Brody (1969) para evaluar las actividades instrumentales de la vida diaria (AIVD). Este cuestionario se utiliza para medir las habilidades de vida independiente de un individuo. Hay tres actividades en la cultura occidental que se consideran pertenecientes en su mayoría a las mujeres, sobre todo en los grupos de edad de este estudio de investigación: la preparación de alimentos, limpieza, lavado de ropa. Por esta razón, los autores sugirieron excluir estas actividades de evaluación de los hombres. Por lo tanto, las puntuaciones de menos de 5 para los hombres y menos de 8 para las mujeres se consideraron como normales. El declive de las actividades instrumentales evaluados por este índice es predictivo de la disminución de las actividades básicas durante el ingreso hospitalario, y es una razón por la cual algunos autores han propuesto como un indicador de fragilidad (Sager, 1996).

La evaluación cognitiva se realizó con Fototest (Carnero-Pardo y Montoro, 2004). Se trata de una herramienta que tiene la ventaja de ser breve, aplicable a personas sin estudios, ya que ofrece resultados que no están influenciados por el nivel educativo. También utilizamos el cuestionario a informantes sobre el deterioro cognitivo en los ancianos (IQCODE; Morales, González, Delser y Bermejo, 1992). Este cuestionario recoge la opinión de un informador cercano al paciente, preferiblemente su cuidador principal, respecto a los cambios en su situación cognitiva. En las instrucciones dadas al 
informante para llevar a cabo la IQCODE, se les pide que recuerden cuál era su relación antes de la admisión al hospital, y su estado en comparación con su situación actual.

\section{Diseño y procedimiento}

En este trabajo se utilizó un diseño cuasi-experimental de medidas repetidas. Las variables dependientes fueron las mediciones obtenidas por los instrumentos anteriormente descritos. Además, tomando como referencia Baztán et al. (2004), que también se utiliza como variables dependientes la ganancia funcional al alta (calculado como la diferencia entre el índice de Barthel obtenido en casa y el índice de Barthel al alta hospitalaria) y la pérdida funcional (diferencia entre el índice de Barthel al alta y el anterior Índice de Barthel). Se realiza una visita al paciente el día de ingreso y una segunda visita al sexto día de la admisión. Se establece como periodo mínimo de ingreso hospitalario 5 días, ya que según Baztán et al. (2004) en un estudio sobre la ganancia funcional y la duración de la estancia en el hospital, establece que para el cálculo de la ganancia funcional la estancia tenía que ser entre 5 y 9 días. Se excluyeron aquellos pacientes que permanecieron menos de cinco días en la unidad. Por último, entre los 60 y 90 días después del alta hospitalaria se realiza una tercera visita en el domicilio. El tiempo establecido para llevar a cabo esta visita se basa en la presencia de un pico de ganancia funcional que se produce en la semana octava en el estudio de Baztán et al. (2004).Por otra parte, debido a la fragilidad del participante que no era posible extender este tiempo más allá de 90 días, ya que es posible que otra patología o el agravamiento podría interferir con los resultados. El análisis se realizó con el paquete estadístico SPSS, versión 19.0 para Windows.

\section{RESULTADOS}

\section{Dependencia funcional}

Utilizando el Índice de Barthel, que mide la dependencia funcional al ingreso $(M=36.47, S D=24.73)$, y al alta hospitalaria $(M=46.69, S D=24.35)$ y posteriormente en el domicilio $(M=67.97, S D=26.12)$. También, a través de los datos aportados por el informador, por lo general el cuidador principal, se estableció el Índice de Barthel previo al ingreso $(M=80.03, S D=23.13)$. Con el fin de verificar si existen diferencias significativas en los valores obtenidos, se realizó un análisis de las varianzas de los valores repetidos. El resultado del análisis mostró la existencia de diferencias significativas, $\quad F_{(3 / 234)}=412.850 ; \quad M S E=87741.441, \quad p^{<0.0001,} \quad \eta \_p^{\wedge} 2=0.640 . \quad$ Las comparaciones posteriores realizadas utilizando la prueba de Scheffé, revelaron que las diferencias están dentro de los cuatro valores del Índice de Barthel $(p<0.001)$, mostrando que hay una disminución en el ingreso, una leve recuperación al alta hospitalaria, que continúa posteriormente en el domicilio, pero sin una recuperación completa de la 
situación de la funcionalidad anterior al incidente que provocó la hospitalización. Además, hemos completado un análisis de las actividades instrumentales utilizando la escala de AIVD de Lawton, tanto antes de la admisión $(M=4.52, S D=2.99)$ y en el domicilio $(M=3.39, S D=3.00)$, con el fin de observar su evolución después de la hospitalización. El análisis de varianza de medidas repetidas indicó la existencia de diferencias significativas en las mediciones realizadas antes y después del ingreso en el hospital, $\left.F_{(1 / 234)}=111.980 ; M S E=1.583, p<0.0001, \eta \_p^{\wedge} 2=0.325\right)$.

Tabla 1. Estadísticos descriptivos de niveles de dependencia

\begin{tabular}{lrrrrrrrr}
\hline \multirow{2}{*}{ Nivel dependencia } & \multicolumn{2}{c}{ Antes del ingreso } & \multicolumn{2}{c}{ En el ingreso } & \multicolumn{2}{c}{ Al alta } & \multicolumn{2}{c}{ Posterior al alta } \\
\cline { 2 - 10 } & Frecuencia & \multicolumn{1}{c}{$\%$} & Frecuencia & $\%$ & Frecuencia & $\%$ & \multicolumn{1}{c}{ Frecuencia } & $\%$ \\
\hline Dependientes altos/severos & 40 & 15.74 & 204 & 80.31 & 174 & 68.5 & 78 & 33.3 \\
\hline Dependientes leves/moderados & 127 & 50.01 & 45 & 17.71 & 77 & 30.3 & 115 & 49.2 \\
\hline Independientes & 87 & 34.25 & 5 & 1.96 & 3 & 1.18 & 41 & 17.5 \\
\hline Total & 254 & 100 & 254 & 100 & 254 & 100 & 234 & 100 \\
\hline
\end{tabular}

\section{El deterioro cognitivo}

Se evaluó la función cognitiva con Fototest (Sager, 1996). Esta prueba se llevó a cabo en la admisión hospitalaria $(M=27.28, S D=8.616)$ y en la visita posterior al alta en el domicilio $(M=28.75, S D=9.501)$. El análisis de varianza de medidas repetidas mostró la existencia de diferencias significativas entre las dos etapas de deterioro cognitivo, $F_{(1 / 234)}=4.818 ; M S E=12.162, p<0.029, \eta \_p^{\wedge} 2=0.022$. Con el fin de saber si se habían producido cambios en la funcionalidad cognitiva entre los períodos anteriores a la admisión y el alta, de hecho para saber si el paciente anciano había sufrido una disminución en su función cognitiva debido al proceso de hospitalización, se utilizaron como variables los resultados de la IQCODE. Sólo se pudo obtener una puntuación de 229 pacientes ya que el resto de los pacientes de edad avanzada estaban solos durante las visitas en el domicilio y no había ningún cuidador principal que podría llegar a ser el informador. Sólo un $6.55 \%$ de los entrevistados pensaban que su pariente había tenido algún tipo de recuperación después de la admisión, por lo que respecta a la situación anterior, $43.66 \%$ pensaba que no había cambiado en absoluto, y 49.35\% consideró que había empeorado en relación al estado cognitivo que tenía antes del ingreso.

Tabla 2. Estadísticos descriptivos de puntuaciones del fototest

\begin{tabular}{lccccc}
\hline & $N$ & Mínimo & Máximo & $\bar{X}$ & $S_{x}$ \\
\hline FOTOTEST AL INGRESO & 254 & 0 & 46 & 27.28 & 8.616 \\
FOTOTEST DOMICILIO & 234 & 0 & 47 & 28.75 & 9.501 \\
\hline
\end{tabular}

Relación entre la dependencia funcional y el deterioro cognitivo con características sociodemográficas

Género:

Con el fin de verificar si existe una incidencia diferencial de género de declive funcional, se realizó un análisis de varianza utilizando la variable género como factor y 
como variables dependientes los diversos resultados obtenidos. Los resultados mostraron la existencia de diferencias significativas en la dependencia y los índices de deterioro cognitivo de los hombres y las mujeres. En concreto, las diferencias en la Escala de Lawton en el hogar, el Índice de Barthel en el hogar y la IQCODE. Se observó que las mujeres tuvieron una menor dependencia funcional en el domicilio y también, de acuerdo con los informadores, su condición había empeorado menos en relación con el período anterior a la admisión hospitalaria. No se encontraron diferencias de género en los valores del anterior Índice de Barthel, ni al ingreso ni al alta, y de hecho, durante la hospitalización. Sin embargo, como podemos ver en la figura 1, las mujeres siempre puntuaron más alto que los hombres.

Tabla 3. Estadísticos descriptivos y resultados del ANOVA para los índices de dependencia y deterioro cognitivo en función del sexo

\begin{tabular}{|c|c|c|c|c|c|c|c|}
\hline & \multicolumn{2}{|c|}{ Mujeres } & \multicolumn{2}{|c|}{ Hombres } & \multirow{2}{*}{ Media cuadrática } & \multirow{2}{*}{$F^{a}$} & \multirow{2}{*}{$d$} \\
\hline & $\bar{x}$ & $S_{x}$ & $\bar{X}$ & $S_{x}$ & & & \\
\hline Barthel previo & 81.085 & 22.687 & 76.129 & 24.531 & 1044.100 & 1.959 & 0.214 \\
\hline Barthel ingreso & 37.70 & 25.988 & 31.91 & 18.882 & 1428.958 & 2.348 & 0.234 \\
\hline Barthel alta & 47.42 & 24.428 & 44.02 & 24.123 & 489.286 & 0.824 & 0.139 \\
\hline Bartel domicilio & 70.452 & 24.555 & 57.826 & 29.957 & 5891.613 & $8.925 * *$ & 0.491 \\
\hline Lawton ingreso & 4.69 & 3.041 & 3.91 & 2.728 & 26.041 & 2.936 & 0.079 \\
\hline Lawton domicilio & 3.64 & 3.046 & 2.37 & 2.594 & 59.989 & $6.830 * *$ & 0.266 \\
\hline Fototest ingreso & 27.43 & 8.719 & 26.74 & 8.297 & 20.156 & 0.271 & 0.260 \\
\hline Fototest domicilio & 29.29 & 9.247 & 26.76 & 10.261 & 227.215 & 2.535 & 0.428 \\
\hline IQCODE & 3.52 & 0.881 & 4.00 & 0.935 & 8.988 & 11.287 *** & 0.535 \\
\hline
\end{tabular}

Figura 2. Evolución del índice de Barthel en función del género

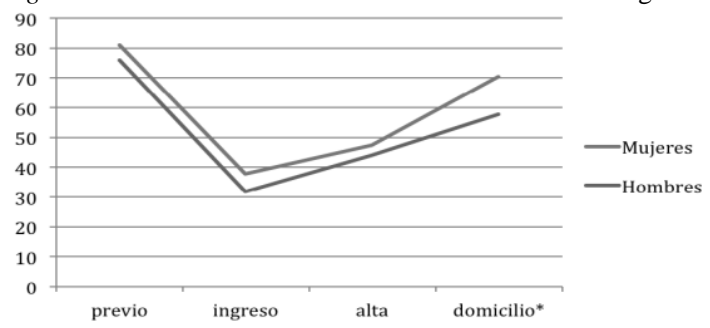

Por otra parte, se utilizó una tabla de contingencia para correlacionar los niveles de los participantes de la dependencia, al ingreso y al alta, con el género. La tipificado corregido residuos de la tabla de contingencia indican que en el momento de la admisión, en el grupo de nivel "alto de dependencia/grave", hay una proporción significativamente mayor de hombres $(92.5 \%)$ que las mujeres $(77.5 \%)$.

Edad:

Edad muestra una significativa asociación lineal inversa $(p<0.01)$ con toda la dependencia o las variables indicativas descenso: Índice de Barthel al ingreso 
( $r x y=-0.497)$, el Índice de Barthel al alta $(r x y=-0.557)$, Lawton anterior $(r x y=-0.633) \mathrm{y}$ Fototest al ingreso $(r x y=-0.441)$. Es decir, mientras mayores son los pacientes más baja es la puntuación de los indicadores de dependencia, y es mayor el deterioro cognitivo.

Tabla 4. Prueba $t$ de Student para los índices dependencia y deterioro cognitivo en función de la edad

\begin{tabular}{lcccccc}
\hline & \multicolumn{2}{c}{ De 65 a 80 años } & \multicolumn{2}{c}{$\geq 81$ años } & \multirow{2}{*}{$t$} & \multirow{2}{*}{$D$} \\
\cline { 2 - 5 } & $\bar{X}$ & $S_{X}$ & $\overline{\bar{X}}$ & $S_{X}$ & & \\
\hline Barthel previo & 80.76 & 17.23 & 69.82 & 24.16 & $-7.602 * *$ & 0.948 \\
\hline Barthel al ingreso & 46.25 & 26.31 & 26.31 & 18.08 & $-7.018^{* * *}$ & 0.883 \\
\hline Barthel al alta & 58.54 & 23.36 & 34.35 & 18.61 & $-9.050^{* *}$ & 0.497 \\
\hline Barthel domicilio & 79.67 & 25.43 & 55.43 & 20.50 & $-7.994 * *$ & 1.140 \\
\hline Fototest ingreso & 30.11 & 8.655 & 24.46 & 7.621 & $-5.434 * *$ & 0.691 \\
\hline Fototest domicilio & 31.94 & 9.673 & 25.35 & 8.063 & $-5.379 * *$ & 0.738 \\
\hline **Nivel de significación, $p<.01$ en todas las variables. & & &
\end{tabular}

Estado civil:

Descubrimos una relación significativa $(p<0.01)$ entre el estado civil y el nivel de dependencia de todos los indicadores cognitivos y funcionalidad: Índice de Barthel previo a la hospitalización o el ingreso ( $r s=-0.263)$, Índice de Barthel al alta $(r s=-0.258)$; Barthel Índice en el hogar ( $r s=-0.196)$, Escala de Lawton en el ingreso ( $r s=-0.356)$, Escala de Lawton en casa ( $r s=-0.307)$; Fototest al ingreso ( $r s=-0.189)$ y Fototest en casa $(r s=-0.173)$.

Como se puede observar en la figura 3, la evolución del índice de Barthel sigue la misma tendencia general, independientemente del estado civil del paciente de edad avanzada. Hay una repentina caída en el ingreso con una ligera recuperación en el alta, que sigue en casa, pero sin llegar a las cifras de antes de la admisión. También se observa que hay diferencias entre los pacientes casados, en relación con las mujeres viudas, ya que su recuperación es más rápida y mayor en el alta y en casa. Una tendencia similar se observó cuando se analizó la Escala de Lawton y Fototest.

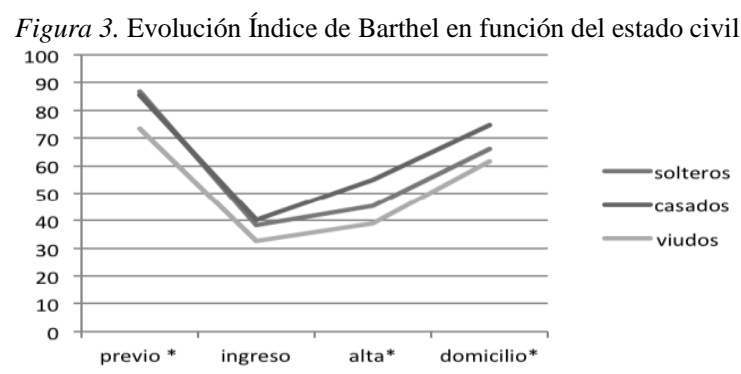

El análisis unifactorial de la varianza de estas mediciones mostró la existencia de diferencias significativas en relación con el estado civil, el nivel de dependencia funcional medida por el Índice de Barthel (ADL), medido por la Escala de Lawton (AIVD), y la funcionalidad cognitiva medida por Fototest. Comparaciones post hoc, 
utilizando la prueba de Scheffé, reveló que las diferencias encontradas están presentes en todas las variables medidas, entre los grupos de casados y de viudas, en lo que respecta al hecho de que los pacientes ancianos casados eran más independientes.

Si se analizan las categorías de los diferentes niveles de dependencia funcional y el deterioro cognitivo en relación con el estado civil y las tablas de contingencia correspondientes nos damos cuenta de que, durante el período previo al ingreso hospitalario, en el grupo de mujeres casadas existe una proporción significativamente menor de la esperada según la hipótesis de independencia, $(p<0.01)$ de pacientes ancianos con graves/alta dependencia $(8.1 \%)$ y baja/moderada dependencia $(42.3 \%)$ y hay una proporción significativamente mayor de pacientes casadas en el grupo de los independientes $(49.54 \%)$. También en el grupo de viudas hay una proporción significativamente mayor $(p<0.01)$ de pacientes con dependencias de cualquier nivel ( $23.14 \%$ con dependencia alta, el $58.6 \%$ con dependencia leve) y una proporción significativamente menor de independientes viudos (18.1\%). Asimismo, en la evaluación del estado cognitivo al ingreso, realizada con Fototest, se encontró una proporción significativamente mayor $(p<0.01)$ de los sujetos casados sin deterioro cognitivo, de lo que se esperaba, $(53.5 \%)$ y una proporción significativamente mayor de viudas pacientes con deterioro cognitivo $(68.3 \%)$.

Por otro lado, y con el fin de analizar las diferencias en la variable de ganancia hospital en relación con el sexo y el estado civil, se realizó un ANOVA (2x3). El resultado muestra que existen diferencias significativas según el estado civil, $F_{(2 / 245)}=2.714 ; \quad M S E=346.126, \quad p<0.05, \eta \_p^{\wedge} 2=0.269$, pero se muestran diferencias significativas en relación al género, o a la interacción entre ambos factores. Comparaciones post hoc revelaron que las diferencias significativas fueron en el grupo casada que mostraron una mayor ganancia funcional en el hospital $(M=14.45, S D=13.41)$ y la viuda con una ganancia inferior $(M=5.82, S D=20.57)$. El grupo de solteros $(M=7.14$, $S D=27.44)$ no mostró diferencias significativas en relación a los otros grupos.

Nivel educativo:

El nivel educativo se relacionó significativamente $(p<0.01)$ en forma directa con el Índice de Barthel: anterior $(r s=0.278)$, al ingreso $(r s=0.251)$ y al alta $(r s=0.296)$, y también con la Escala de Lawton anterior al ingreso, $(r s=0.296)$, Fototest al ingreso $(r s=0.190)$. Es decir, los pacientes con mayor nivel educativo tenían mayores puntuaciones en los índices de Barthel, Lawton y Fototest, por tanto, menor deterioro cognitivo y funcional y un nivel de dependencia menor. Sin embargo, el nivel educativo no presenta una relación significativa con el aumento de deterioro funcional. Un ANOVA unifactorial mostró la existencia de diferencias significativas en el nivel de dependencia, en relación con el nivel educativo: el grupo de pacientes con educación mostraron puntuaciones más altas en todas las variables funcionales de dependencia. También se encontraron diferencias significativas en el deterioro cognitivo en relación 
con el nivel educativo: los pacientes con un determinado nivel educativo tienen mejores resultados en los indicadores de deterioro cognitivo que aquellos sin ninguna educación.

Tabla 5. ANOVA indicadores de dependencia funcional en función del nivel de estudios

\begin{tabular}{lrrrrrrrr}
\hline \multirow{2}{*}{ Variables dependientes } & \multicolumn{2}{c}{ Sin estudios } & \multicolumn{2}{c}{ Con estudios } & \multirow{2}{*}{ MC } & \multirow{2}{*}{$F_{(1 / 254)}$} & \multirow{2}{*}{$d$} \\
\cline { 2 - 6 } & $\bar{X}$ & $S_{X}$ & $\bar{X}$ & \multicolumn{1}{c}{$S_{X}$} & & \\
\hline I.Barthel Previo & 76.08 & 23.35 & 86.97 & 21.10 & 6961.001 & $13.661^{* *}$ & -0.489 \\
\hline I.Barthel Ingreso & 32.43 & 24.25 & 43.64 & 24.07 & 7392.483 & $12.637^{* *}$ & -0.463 \\
\hline I.Barthel Alta & 41.37 & 24.04 & 55.87 & 22.15 & 12250.594 & $22.423^{* *}$ & -0.626 \\
\hline I.Lawton Ingreso & 3.86 & 2.934 & 5.70 & 2.720 & 198.145 & $24.201^{* *}$ & -0.649 \\
\hline Fototest Ingreso & 26.17 & 8.756 & 29.39 & 7.978 & 576.986 & $7.993^{* *}$ & -0.383 \\
\hline
\end{tabular}

\section{DISCUSIÓN Y CONCLUSIONES}

Los resultados de este estudio muestran que los pacientes de edad avanzada aumentan su nivel de dependencia durante el ingreso hospitalario, y logran una ligera recuperación en el alta, pero sin llegar a la dependencia de los valores previos a la admisión. Por lo tanto, confirman los hallazgos de otros estudios similares: DelgadoParada et al. (2009), Formiga y Soto (2009), Mañas et al. (2005) y Valderrama y Molpeceres (1998), que afirman que hay una pérdida de la función, (en particular, Delgado-Parada afirma que es mayor de 12 puntos de acuerdo con el Índice de Barthel), que no se recuperará antes de tres meses.

En el caso de deterioro cognitivo, evaluado por la prueba del informador o cuidador principal del paciente anciano en casa, hemos comprobado que sólo el $5.8 \%$ ha observado recuperación del estado cognitivo después del ingreso en relación a su condición anterior, el $43.6 \%$ confirmó que su estado cognitivo se ha agravado durante el ingreso y el $38.6 \%$ no se ha observado ningún cambio en absoluto. El empeoramiento del estado cognitivo puede ser debido a la inmovilización durante la hospitalización (Calero-García, 2012; Varela et al., 2004a; Varela et al., 2004b). En cuanto a la relación entre los indicadores utilizados para evaluar la dependencia funcional y el deterioro cognitivo con el género, nuestros resultados son similares a los de Carnero et al. (2007). Hay una incidencia diferencial de deterioro funcional en relación con el género, las mujeres tienen una dependencia funcional significativamente menor en el hogar que los hombres $\mathrm{y}$, según sus cuidadores principales, no empeoren tanto como los hombres respecto al período anterior al ingreso hospitalario. Sin embargo, estos resultados no concuerdan con el estudio realizado por Varela et al. (2004b), que encontró una relación entre el deterioro cognitivo y de género. Esta discrepancia puede explicarse por el hecho de que estos autores evaluar el nivel de deterioro antes de la hospitalización y las diferencias encontradas en nuestro estudio se estableció en el período después del alta hospitalaria. Por otro lado, hemos encontrado importantes relaciones inversas entre los diversos niveles de dependencia y el deterioro cognitivo y la edad de los pacientes. De 
acuerdo con otros estudios (Covinky y Palmer, 2003; Varela et al., 2004a), nuestros datos indican que a mayor edad, mayor es la dependencia funcional y el deterioro cognitivo. Además, los resultados evidencian la existencia de diferencias, entre las edades de 65 a 80 años de edad y el grupo de más de 80 años de edad, tanto en la funcionalidad cognitiva y los indicadores de dependencia: siendo las de los pacientes mayores de 80 años significativamente peores. También encontramos variaciones en todos los índices aplicados como con el estado civil del paciente. El análisis mostró que los pacientes casados difieren significativamente de las viudos: los primeros son más independientes y mostraron menor deterioro cognitivo.

En cuanto al nivel educativo, se ha encontrado que los pacientes con un mayor nivel educativo tienen mejores puntajes en el Índice de Barthel, Lawton y Fototest.

Como conclusión, podemos resumir los resultados de este estudio al afirmar de los pacientes mayores de 65 años de edad que son propensas a sufrir un mayor deterioro funcional y cognitivo, durante el ingreso hospitalario en hospital de agudos, son los mayores de 80 años de edad, viudos y sin educación. Además, y de acuerdo con el análisis de las variaciones de nivel de la dependencia y de deterioro cognitivo, es evidente el paciente anciano debe ser hospitalizado sólo durante el período de tiempo imprescindible, y nunca con estancias superiores a quince días.

\section{REFERENCIAS}

Baztán, J.J., Fernandez-Alonso, M., Aguado, R. y Socorro, A. (2004). Resultados al año de la rehabilitación tras fractura de fémur proximal. Anales de Medicina Interna, 21(9), 25-32.

Bravo, G. (2008). Valoración Funcional. En F. Guillen, J. Pérez del Molino y R. Petidier (Eds.), Síndromes y cuidados del paciente geriátrico (pp. 233-238). Barcelona: Elservier-Masson.

Calero, D. y Navarro, E. (2011). Differences in cognitive performance, level of dependency and quality of life (QoL), related to age and cognitive status in asample of Spanish old adults under and over 80 years of age. Archives of Gerontology and Geriatrics, (53), 292-297.

Calero, M.D., Navarro, E., Gómez, A., Lopez, I. y Calero-García, M.J. (2008). Olvidos y memoria: Relaciones entre memoria objetiva y subjetiva en la vejez. Revista Española de Geriatría y Gerontología, 4(43), 299-307.

Calero-García, M.J. (2008). De la discapacidad a la dependencia: Aspectos sanitarios. Seminario Médico, 60(2), 93-100.

Calero-García, M.J., Ortega, A.R., Navarro, E., Jiménez, C. y Calero, D. (2011). Impact of admissions for bone fractures on the dependency ratio of adults over 65 years of age in southern Spain. Archives of Gerontology and Geriatrics. doi: 10.1016/j.archger.2011.08.017.

Canga, A. y Narvaiza, M. (2006). Intervención de enfermería para disminuir las complicaciones derivadas de la hospitalización en los ancianos. Gerokomos, 17(1), 24-31.

Carnero-Pardo, C., Sáez-Zea, C., Montiel, L., Saz, P., Feria, I., Pérez, M.J.R. et al. (2007). Utilidad diagnóstica del test de las fotos (Fototest) en deterioro cognitivo y demencia. Revista De Neurología, 22(10), 860-869. 
Carnero-Pardo, C. y Montoro-Ríos, M.T. (2004). Test de las fotos. Revista De Neurología, 1(39), 801-806.

Covinsky, K.E. y Palmer, R.M. (2003). Loss of independence in activities ofdaily living in older adults hospitalized with medical illnesses: Increased vulnerability with age. Journal of the American Geriatrics Society, (51), 451-458.

Cruz, A., Pancorbo, P., García, F., Carrascosa, M.I., Jiménez, M.C. y Villar, R. (2010).Deterioro funcional en ancianos ingresados en un hospital de agudos sin unidades geriátricas. Gerokomos, 21(1), 8-16.

Delgado-Parada, E., Suarez, F.N., Miniana, J.C., Medina, A., López-Gaona, V., Gutiérrez, S. et al. (2009). Variables asociadas al deterioro funcional al alta y al los tres meses, en ancianos hospitalizados por insuficiencia cardiaca. Revista Española de Geriatría y Gerontología, 2(44), 66-72.

Elipe, M.P. (2006). Condicionantes afectivos de fragilidad en personas mayores. Tesis Doctoral. Universidad de Granada, Granada.

Feng, L., Scherer, S., Tan, B., Chan, G., Fong, N. y Ng, T. (2010). Comorbid cognitive impairment and depression is a significant predictor of poor outcomes in hip fracture rehabilitation. International Psychogeriatrics, 22(2), 246.

Formiga, F. y Soto, A. (2009). Characteristics of falls-related hip fracture in institutionalized elderly patients. The Journals of Gerontology, 64A(9), 992.

Granger, C.V., Dewis, L.S., Peters, N.C., Sherwood, C.C. y Barrett, J.E. (1979). Stroke rehabilitation: Analysis of repeated Barthel index measures. Archives of Physical Medicine Rehabilitation, 1(60), 14-17.

Gutiérrez, J., Domínguez, V. y Solano, J.J. (1999). Deterioro funcional secundario a la hospitalización por enfermedad aguda en el anciano. Análisis de la incidencia y de los factores asociados. Revista Clínica Española, 1(199), 418-423.

Lawton, M.P. y Brody, E.M. (1969). Assessment of older people: Self-maintaining and instrumental activities daily living. Gerontologist, 9(3), 179-186.

Macias, M.C., Guerrero, M.T. y Hernández, V. (2008). Valoración Mental. In F. Guillen Llera, J. Perez del Molino y R. Petidier (Eds.), Síndromes y cuidados del paciente Geriátrico. (pp. 245-260). Barcelona: Elsevier-Masson.

Mañas, M.D., Marchan, E., Conde, C., Sánchez, S., Sánchez-Maroto, T. y Molina, M.C. (2005). Deterioro de la capacidad funcional en pacientes ancianos ingresados en un Servicio de Medicina Interna. Anales de Medicina Interna, 22(3), 131,132.

Martín, I., Gorroñogoitia, A., Gómez, J., Baztán, J. y Abizanda, P. (2010). El anciano frágil. Detección y tratamiento en AP. Atención Primaria, 42(7), 388-393.

Morales, J.M., González, J.L., Delser, T. y Bermejo, F. (1992). Estudio de validación del S-IQCODE: La versión española del Informant Questionnaire on Cognitive Declined in Elderly. Archivos de Neurobiología, 55(6), 262-266.

Navarro, E., Calero, D., López, A., Gómez, A., Torres, I. y Calero-García, M.J. (2008). Nivel de dependencia en la vida diaria y plasticidad cognitiva en la vejez. Escritos de Psicología, 2(1), 74-84.

Pedone, C., Ercolani, S., Catani, M. y Maggio, D. (2005). Elderly patients with cognitiveimpairment have a high risk for functional decline during hospitalization: The GIFA study. The Journals of Gerontology, 60A(12), 15-76.

Sager, M.A., Rudberg, M.A., Jalaluddin, M., Franke, T., Inouye, S. y Landefeld, S. (1996). Hospital admission risk profile (HARP): Identifying older patients at riks for functional decline following acute medical illness and hospitalization. Journal American Geriatric Society, (44), 251-257. 
Shah, S., Vanclay, F. y Cooper, B. (1989). Improving the sensitivity of the Barthel Index for stroke rehabilitation. Journal Clinical Epidemiology, 42, 703-709.

Valderrama, E. y Molpeceres, J. (1998). Seguimiento a largo plazo de los pacientes ingresados en una unidad de media estancia o convalecencia. Revista Española de Geriatría y Gerontología, 1(33), 67-72.

Varela, L., Chávez, H., Gálvez, M. y Méndez, F. (2004a). Características del deterioro cognitivo en el adulto mayor hospitalizado a nivel nacional. Revista de la Sociedad Peruana de Medicina Interna, 17(2), 37-42.

Varela, L., Chávez, H., Herrera, A., Ortiz, P. y Chigne, O. (2004b). Valoración geriátrica integral en adultos mayores hospitalizados a nivel nacional. Diagnóstico, 43(2), 57-63.

Villón, J.M. y Pecho, L.A. (2003). Valoración geriatrica integral y aspectos perioperatorios en ancianos hospitalizados con fractura de cadera por caídas (pp. 24-33). Lima-Perú: Unpublished 1, Universidad Nacional Mayor de San Marcos.

Recibido: 10 de mayo de 2013

Recepción Modificaciones: 24 de junio de 2013

Aceptado: 18 de septiembre de 2013 